\title{
PERFIL ANTROPOMÉTRICO DE LOS PAÍSES PARTICIPANTES DEL CAMPEONATO SUDAMERICANO JUVENIL FEMENINO DE FÚTBOL AÑO 2006.
}

\section{ANTHROPOMETRIC PROFILE OF THE PARTICIPANTS IN THE SOUTH AMERICAN CUP YOUNG WOMEN OF FOOTBALL SOCCER 2006}

\author{
Soto Silva, Víctor Hugo ${ }^{1,2}$ \& Huerta Ojeda, Álvaro Cristian ${ }^{1}$ \\ ${ }^{1}$ Escuela Educación, Carrera de Educación Física, Universidad Santo Tomás. \\ ${ }^{2}$ Escuela Educación Física, Pontificia Universidad Católica de Valparaíso.
}

SOTO, V. \& HUERTA, A. (2013). Perfil antropométrico de los países participantes del campeonato sudamericano juvenil femenino de fútbol año 2006. Mot. Hum. 14(1): 29-35.

\section{RESUMEN}

El propósito de este estudio es determinar el perfil antropométrico de los países participantes del campeonato sudamericano juvenil femenino, realizado en Chile en enero de 2006. La investigación es del tipo cuantitativo con un alcance Exploratorio Descriptivo del tipo No Experimental Transeccional, la muestra es de 120 jugadoras de seis de los nueve países participantes. Los datos se analizarán con medidas de tendencia central, incorporando parámetros antropométricos básico (peso, talla e IMC) y Somatotipo por el método de Heath \& Carter. Los sujetos evaluados

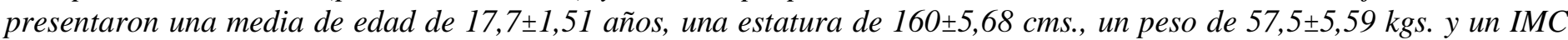
de $22,2 \pm 1,77 \mathrm{~kg} / \mathrm{m}^{2}$. El somatotipo general de las deportistas participantes de esta investigación fue endo-mesomorfo (4,7- 4,2-2,0). Las participantes del Subamericano Juvenil Femenino de Fútbol, realizado en Chile en enero de 2006, presentan un elevado componente graso $(4,7)$, seguido por un componente muscular en el límite inferior de parámetros normales $(4,2)$ y de tejido óseo levemente bajo para deportistas $(2,0)$.

Palabras claves: Somatotipo, Perfil Antropométrico, Fútbol Femenino.

\begin{abstract}
The purpose of this study was to determine the anthropometric profile of the participating countries in south american youth female championship football held in Chile in January 2006. This is type quantitative descriptive and exploratory scope non experimental trans sign players is 120 of ten participating countries. Data will be analyzed with measures of central tendency, incorporating basic anthropometric parameters (weight, height, BMI) and somatotype by the method of

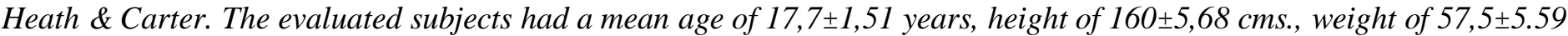
$\mathrm{kgs.}$, and a BIM of 22,2 $\pm 1,77 \mathrm{~kg} / \mathrm{m}^{2}$. General somatotype athletes praticipating of this research was endo-mesomorph (4,7-4,2-2,0). Participants of south american championship football youth female of 2006 have a high fat component (4.7), followed by a muscular component at the lower limit of the normal range (4.2) and bone tissue just under for athletes (2.0).
\end{abstract}

Key Words: Somatotype, Anthropometric Profile, Women's Football. 


\section{INTRODUCCIÓN}

Hoy en día, las Ciencias de la Actividad Física no pueden prescindir de otras ciencias para apoyarse. El uso de otras disciplinas brindan la oportunidad de tener una perspectiva distinta de las actividades que llevamos a cabo, además, bien utilizadas pueden ser de gran utilidad para un mejor desarrollo del deporte y la Educación Física.

Una de las áreas en constante desarrollo corresponde a las evaluaciones físico - deportivas. Éstas contemplan protocolos con distintos grados de complejidad, y se dividen según criterios de validez, objetividad, confiabilidad y factibilidad.

Dentro de las mediciones corporales, existe el registro de las mediciones antropométricas. Éstas pueden ser acompañadas con diferentes programas computacionales, determinando parcial o totalmente algunas de las variables morfológicas de la estructura humana.

La antropometría, es un método que entre otras aplicaciones, se ha sido utilizado para describir y comparar deportistas en distintos niveles de competencia; para caracterizar los cambios físicos durante el crecimiento, el envejecimiento, y el entrenamiento; para comparar las formas relativas de hombres y mujeres; y como herramienta de análisis de la "imagen corporal" (Norton y Olds, 1996).

Muchos de los estudios antropométricos realizados en poblaciones de deportistas, han destacado que el perfil antropométrico es un factor selectivo importante para el éxito de cada atleta o equipo en sus respectivos deportes. Los datos más importantes que se han observado, dentro de este contexto, han sido a través de las mediciones y estimaciones realizadas a través del somatotipo.

El somatotipo determinado por el método de Carter y Heath (Carter y Heath, 1990), es el más utilizado para el análisis del físico de los deportistas, definiéndose como la cuantificación de la forma y composición corporal presente en una persona a partir de tres numerales; estos números representan los componentes endomórfico (adiposidad relativa), mesomórfico (desarrollo músculo esquelético relativo) y ectomórfico (linealidad relativa), siempre en ese orden, cuantificando el físico de un sujeto como un todo, estableciendo una relación entre la adiposidad, la masa muscular y ósea y la relación de linealidad a través del peso y la talla respectivamente. Existen estudios realizados sobre el análisis del somatotipo de futbolistas profesionales, el cual ha sido determinado en general como mesomorfo balanceados con valores en endomorfia (2-3), mesomorfia (4.7-5.3) y ectomorfia (1.9-3), (Carter, 2002; Rienzi y Mazza, 1998; Rienzi et al., 2000; Reilly et al., 2000; Tomkins, Popovic y Martin, 2003). Si bien son varios los estudios realizados a futbolistas hombres, no ocurre lo mismo con respecto a investigaciones que se hayan hecho a grupos de mujeres futbolistas.

El estudio morfológico del deportista, a través de la cineantropometría, se ha orientado principalmente desde el análisis antropométrico por variable, la composición corporal, el somatotipo y la proporcionalidad. Según Rivera J., (Rivera, 2006) en diversos estudios, se ha visto como la morfología de deportistas presenta un perfil antropométrico con diferencias en diversas variables, principalmente en la estatura, masa corporal, pliegues y algunas circunferencias, tanto por deporte como por posiciones de juego, diferencias físicas identificadas tanto en deportistas de alto rendimiento como en aquellos de nivel estudiantil-universitario.

Los objetivos de la antropometría son el somatotipo, la forma y la composición corporal. Carter y Heath (Carter y Heath, 1990) basándose en antecedentes aportados por Sheldon, definieron el somatotipo como "la descripción numérica de la configuración morfológica de un individuo en el momento de ser estudiado".

Según Ruiz (Ruiz et al., 2001), el somatotipo, en el deporte, permite conocer el estado físico de una población, comparar los deportistas de diferentes especialidades y sexos, y señalar la tendencia adecuada para cada individuo, determinando el sentido de su desarrollo. 


\section{MATERIAL Y MÉTODO}

\section{Enfoque, Alcance y Diseño de Estudio.}

El estudio es del tipo cuantitativo, caracterizado por medir y analizar el fenómeno de "morfología corporal" de manera objetiva utilizando estadística de tendencia central. El alcance es Exploratorio Descriptivo, y el diseño corresponde al tipo No Experimental Transeccional (Hernández, Fernández y Baptista, 2006).

\section{Población.}

La población estudiada corresponde a todas las jugadoras juveniles de los países de Sudamérica que participaron en el campeonato sudamericano de la categoría, desarrollado en la ciudad de Viña del Mar, Chile, en enero del año 2006. Dicha cifra equivale a 200 sujetos.

\section{Muestra.}

La muestra es no probabilística, y está dada por jugadoras de los países que aceptaron ser examinadas, estos fueron Bolivia, Colombia, Ecuador, Paraguay, Uruguay y Venezuela, cada equipo estaba constituido por 20 jugadoras, lo que nos da una muestra de 120 sujetos equivalente al $60 \%$ de la población.

\section{Selección del Instrumento y método de Evaluación.}

El instrumento de evaluación seleccionado para la estimación del somatotipo de las jugadoras de Fútbol participantes del Sudamericano de la especialidad, desarrollado en enero de 2006 en Chile, es la prueba de Somatotipo de Heath y Carter. Éste permite clasificar la muestra dentro de uno los tres tipos de morfología (mesomorfo, endomorfo o ectomorfo).

El método de Heath y Carter ocupa como variables el Peso y la Talla, cuatro Pliegues Cutáneos (Pliegue Tricipital, Pliegue Subescapular, Suprailíaco o Supraespinal y Pantorrilla Media), dos Circunferencias (pantorrilla en posición de pie perímetro máximo- y el Bíceps Braquial contraído), además de dos Diámetros Óseos (distancia Epicondilia Humeral -Epicóndilo y Epitróclea- y el diámetro del Fémur -Cóndilos Femorales-).

\section{Aplicación del Instrumento.}

Una vez que los evaluadores contaron con la autorización del Cuerpo Técnico de los países que participaron del sudamericano de Fútbol juvenil femenino, realizado en Chile durante el mes de enero de 2006, se acordó que todas las mediciones se realizarían antes del comienzo del torneo, un equipo por día en horarios entre las 18 y 20 horas; para tan efecto, se dispuso una habitación habilitada especialmente para la realización del presente estudio, donde se dispusieron los materiales que se detallan a continuación:

Balanza y Estadiómetro:

El Health o Meter Professional tiene números grandes, una plataforma estable y una alta precisión en la medida.

La balanza puede medir hasta las 450 libras o 204 kilogramos.

Posee una base de acero y plataforma con exactitud profesional, un ojo grande para nivelar la plataforma y una viga de equilibrio para la lectura del peso.

Peso del producto 20,4 kilogramos.

El Health o Meter Professional tiene la posibilidad de medir el peso y la estatura simultáneamente, posee una vara que va desde los 60 hasta 214 centímetros de altura.

\section{Antropómetro Pequeño:}

Segmómetro rígido (diseño exclusivo de FAGA S.L.R.).

Construido en aluminio anodizado, rígido, extremadamente exacto y durable, por la rigidez de su construcción.

Nueva regla de aluminio anodizado, de una cara para tomar medidas interiores, en la otra cara para medir desde la caja antropométrica hasta la marca correspondiente, con los números en posición vertical para una lectura más cómoda.

\section{Cinta Antropométrica:}

- El kit antropométrico Faga, posee una cinta antropométrica metálica.

\section{Cáliper Plástico:}

Realizado íntegramente en material plástico semirígido de gran durabilidad.

Resortes probados electrónicamente para ofrecer la tensión adecuada para la medición de los pliegues subcutáneos de tejido adiposo. 
SOTO, V. \& HUERTA, A. (2013). Perfil antropométrico de los países participantes del campeonato sudamericano juvenil femenino de fútbol año 2006. Mot. Hum. 14(1): 29-35.

\section{Análisis Estadístico.}

Los resultados fueron analizados por el programa estadístico Graphpad Instat versión 3.5 (2000). Los datos fueron sometidos a un análisis de tendencia central. Previo a esto, los datos fueron sometidos a la prueba Kolmogorov \& Smirnov (KS) con la finalidad de verificar la normalidad de varianza.

\section{RESULTADOS.}

Tabla I. Resultados antropométricos generales de jugadoras participantes del sudamericano de Fútbol realizado en Chile durante el año 2006.

\begin{tabular}{|c|c|c|c|c|c|c|c|c|c|c|}
\hline \multicolumn{2}{|l|}{ Países. } & $\begin{array}{c}\text { Edad } \\
\text { (años). }\end{array}$ & Peso (kg). & Talla $(\mathrm{cm})$. & IMC. & C.Endo. & C.Meso. & C.Ecto. & $\mathbf{X}$. & Y. \\
\hline \multicolumn{2}{|l|}{ Bolivia. } & $18,0 \pm 1,04$ & $58,8 \pm 6,03$ & $158,9 \pm 6,36$ & $23,2 \pm 1,47$ & $5,4 \pm 1,00$ & $4,8 \pm 0,66$ & $1,5 \pm 0,60$ & $-3,9 \pm 1,48$ & $2,7 \pm 1,69$ \\
\hline \multicolumn{2}{|l|}{ Colombia. } & $17,3 \pm 1,08$ & $58,5 \pm 5,28$ & $163,6 \pm 5,10$ & $21,9 \pm 1,98$ & $4,7 \pm 1,23$ & $4,1 \pm 0,83$ & $2,3 \pm 1,12$ & $-2,4 \pm 2,25$ & $1,3 \pm 1,92$ \\
\hline \multicolumn{2}{|l|}{ Ecuador. } & $17,8 \pm 1,48$ & $55,5 \pm 5,80$ & $161,3 \pm 5,88$ & $21,3 \pm 1,04$ & $4,3 \pm 0,63$ & $3,9 \pm 0,65$ & $2,4 \pm 0,47$ & $-1,9 \pm 0,64$ & $1,0 \pm 1,96$ \\
\hline \multicolumn{2}{|l|}{ Paraguay. } & $16,9 \pm 1,99$ & $55,5 \pm 5,75$ & $160,1 \pm 5,96$ & $21,7 \pm 1,97$ & $4,6 \pm 1,00$ & $4,0 \pm 1,11$ & $2,2 \pm 1,04$ & $-2,4 \pm 1,57$ & $1,2 \pm 2,32$ \\
\hline \multicolumn{2}{|l|}{ Uruguay. } & $17,3 \pm 1,77$ & $57,9 \pm 6,02$ & $160,7 \pm 5,02$ & $22,4 \pm 1,83$ & $4,2 \pm 0,86$ & $4,1 \pm 0,73$ & $1,9 \pm 0,87$ & $-2,3 \pm 1,58$ & $2,1 \pm 1,87$ \\
\hline \multicolumn{2}{|l|}{ Venezuela. } & $18,9 \pm 0,84$ & $57,7 \pm 5,37$ & $160,8 \pm 5,62$ & $22,3 \pm 1,63$ & $4,9 \pm 1,04$ & $4,1 \pm 0,68$ & $1,9 \pm 0,83$ & $-2,9 \pm 1,68$ & $1,4 \pm 1,85$ \\
\hline \multicolumn{2}{|c|}{ PROMEDIOS } & $17,7 \pm 1,51$ & $57,5 \pm 5,59$ & $160,9 \pm 5,68$ & $22,2 \pm 1,77$ & $4,7 \pm 1,04$ & $4,2 \pm 0,82$ & $2,0 \pm 0,90$ & $-2,7 \pm 0,73$ & $1,7 \pm 1,97$ \\
\hline $\begin{array}{l}\text { IMC. } \\
\text { Edad. } \\
\text { C.Endo. } \\
\text { C.Meso. } \\
\text { C.Ecto. X. } \\
\text { Y. }\end{array}$ & $\begin{array}{l}= \\
= \\
= \\
= \\
= \\
=\end{array}$ & $\begin{array}{l}\text { Indice de } \\
\text { en años y } n \\
\text { Componen } \\
\text { Componen } \\
\text { Componen } \\
\text { Coordenad } \\
\text { Coordenad }\end{array}$ & $\begin{array}{l}\text { Corporal. } \\
\text { es. } \\
\text { Endomórfico. } \\
\text { Mesomórfico. } \\
\text { Ectomórfico. } \\
\text { K. }\end{array}$ & & & & & & & \\
\hline
\end{tabular}

Al estar categorizado el sudamericano a nivel juvenil, las muestra no presentó gran dispersión en la edad, sólo resalta Paraguay es así como la selección más joven con 16 años 9 meses, y Venezuela como la más longeva con 18 años 9 meses.

En relación al peso corporal, hubo dos equipos en el rango inferior; tanto Ecuador como Paraguay presentaron una media de 55,5 kilogramos en sus jugadoras. Paralelamente, las jugadoras bolivianas presentaron la mayor masa corporal con $58,8 \pm 6,03$ kilogramos.

Al describir la estatura de la muestra, se destaca la selección de Colombia como la más alta, las jugadoras de este equipo presentaron una estura media de $163,6 \pm 5,10$ centímetros (aproximadamente 3 centímetros sobre la media sudamericana). Por el contrario, las jugadoras bolivianas presentaron la menor talla con $158,9 \pm 6,36$ centímetros.

Pese a que todos los Índices de Masa Corporal (IMC) estuvieron en el rango "normal", se destaca la selección boliviana con el valor más alto $\left(23,2 \pm 1,47 \mathrm{~kg} / \mathrm{m}^{2}\right)$; esta cifra fue esperable considerando que la muestra mencionada fue la que tuvo la mayor masa corporal y la menor estatura. Por el otro lado, aparece Ecuador con el IMC más bajo de muestra evaluada, la cifra alcanza los $21,3 \pm 1,04 \mathrm{~kg} / \mathrm{m}^{2}$.

El componente endomórfico, relacionado con el tejido adiposo que posee cada sujeto, se encuentra con valores de 4,7 $\pm 1,04$; cifra elevada considerando que este valor debiese fluctuar entre 2,0 a 2,5 puntos. Cabe mencionar que la selecciones de Bolivia presenta valor más alto de la muestra $(5,4 \pm 1,00$ puntos); por el contario las selecciones de

Ecuador y Uruguay poseen los valores más bajos para esta variable con $4,3 \pm 0,63$ y $4,2 \pm 0,86$ puntos respectivamente.

El componente mesomórfico, que tiene relación a la cantidad de masa muscular, se encuentra en el límite inferior de los parámetros normales $(4,2 \pm 0,82)$, pero es necesario mencionar que la muestra es una selección deportiva de "alto rendimiento", por tanto esta variable debiese oscilar alrededor del valor 5,0. 
SOTO, V. \& HUERTA, A. (2013). Perfil antropométrico de los países participantes del campeonato sudamericano juvenil femenino de fútbol año 2006. Mot. Hum. 14(1): 29-35.

Resalta la masa muscular presentada por la selección de Bolivia con 4,8 $\pm 0,66$ puntos.

$(2,0 \pm 0,90)$, siendo de 2,4 a 2,7 los valores sugeridos para tal componente. Cabe mencionar que los valores más bajos de los países Sudamericanos son

El componente ectomórfico, que tiene relación con el sistema óseo, se presenta levemente bajo los Bolivia, Uruguay y Venezuela con $1,5,1,9$, y 1,9 puntos respectivamente. parámetros deseados en deportistas damas, esto queda ratificado en el valor promedio encontrado

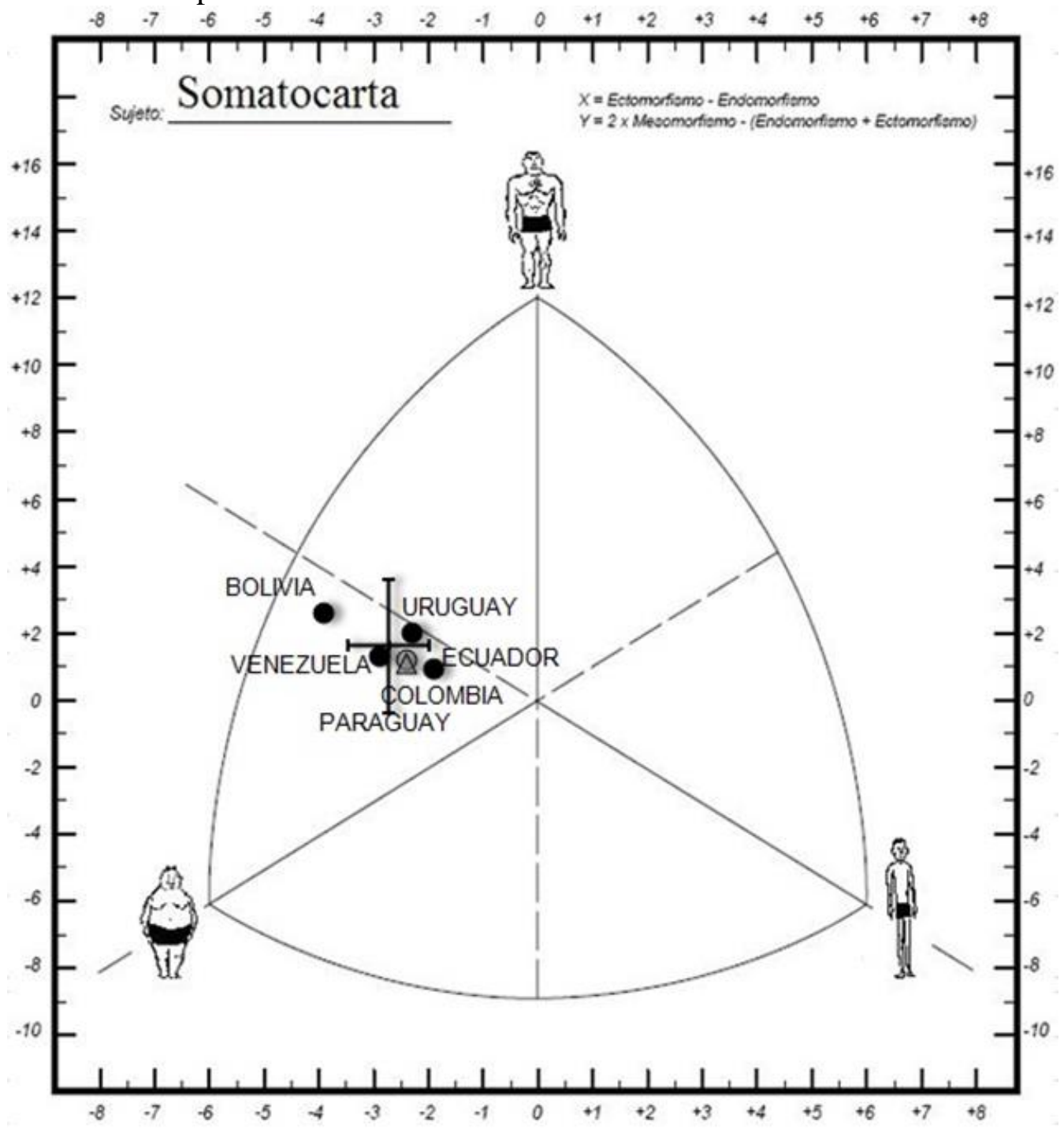

Figura 1. Somatocarta de selecciones de Fútbol participantes del sudamericano de la especialidad, realizado en Chile durante el 2006.

En la Figura 1, se aprecian en forma de coordenanda ( $\mathrm{X}$ e $\mathrm{Y}$ ) los valores tridemensionales medidos y calculados para los componentes meso, endo y ectomórficos.

Los valores medios encontrados para las selecciones que participaron del estudio fueron $-2,7 \pm 0,73 \mathrm{y}$ 1,7 $\pm 1,97$ para $\mathrm{X}$ e $\mathrm{Y}$ respectivamente. De esta media, sólo la selección de Bolivia se aleja más de una desviación estándar de la media, las restantes selecciones se agrupan en torno a los valores descritos.

Los valores medios de la muestra, permiten inferir que las futbolistas que participaron en el campeonato sudamericano de la especialidad en Chile durante el 2006, poseen un componente endomórfico alto para su especialidad y género; un componente mesomórfico en el límite inferior de los valores normales; y un componente ectomórfico bajo. 
SOTO, V. \& HUERTA, A. (2013). Perfil antropométrico de los países participantes del campeonato sudamericano juvenil femenino de fútbol año 2006. Mot. Hum. 14(1): 29-35.

Además, y según la clasificación de Heath \& Carter, la media de muestra quedó clasificada cono endomesomórfíca.

\section{DISCUSIÓN.}

Somatotipo: En ninguno de los países, el valor del ectomorfismo superó a los otros componentes, lo mismo ocurrió en los valores del mesomorfismo que no fueron más que los del endomorfismo.

El somatotipo general de esta investigación, arrojó valores de $(4,7-4,2-2,0)$, al compararlo con el estudio realizado por Can y colaboradores (Can, Yilmaz y Erden, 2004), que fue de $(3,0-3,5-2,4)$ para jugadoras de fútbol profesional, tendríamos que señalar que en el presente estudio los valores fueron mayores en endo y mesomorfismo y más bajo en ectomorfismo, esto se podría atribuir a que las jugadoras profesionales llevan más tiempo dedicado al entrenamiento y a la práctica del deporte, por lo que los valores de endomorfismo son más bajos por aumento del metabolismo, y los valores del mesoforfismo, más altos producto de las adaptaciones musculares a las cargas deportivas; lo anterior también queda ratificado en la evidencia presentada por Malina (1994), donde deja explícito que en "las mujeres deportistas adolescente la adiposidad relativa no se incrementa tanto con la edad como ocurre con las no deportistas". El mismo autor, también menciona que la falta de adiposidad relativa puede retrasar la menarquía, y que la edad esquelética para una misma edad es mayor en las jugadoras de Fútbol que en las mujeres sedentarias.

Pellenc (2006), compara a futbolistas de distinto nivel, concluyendo que a mayor entrenabilidad, menor el es porcentaje graso y mayor es el componente mesomóficos, datos que ratifican la información de este documento .

Como conclusión de los resultados obtenidos en esta investigación en cuanto al somatotipo $(4,7-4,2$ - 2,0), se señala que las características del endomorfismo $(4,7)$ son de adiposidad moderada. Las características del mesomorfismo $(4,2)$ indican que existe un moderado desarrollo muscular. En cuanto al ectomorfismo $(2,0)$, éste indica que hay una linealidad relativa, es decir, gran volumen por unidad de altura.

El somatotipo general de las deportistas participantes de esta investigación fue endo- mesomorfo, donde el endomorfismo es el dominante y el mesomorfismo es mayor que el ectomorfismo.

\section{REFERENCIAS BIBLIOGRÁFICAS.}

Can F, Yilmaz I, Erden Z. (2004). Morphological characteristics and performance variables of women soccer players. The Journal of Strength and Conditioning Research. 18(3):480-485.

Carter, J.E.L., \& Heath, B.H. (1990). Somatotyping development and Aplications. Cambridge. Cambridge University Press.

Carter J. (2002). The Heath \& Carter anthropometric somatotype. Instruction manual. TeP \& Rosscraft. Canada.

Hernández R.; Fernández C. \& Baptista P. (2006). Metodología de la Investigación. $4^{\circ}$ Edición. Editorial McGraw Hill. D.F., México.

Malina, R. (1994). Crecimiento Físico y Maduración Biológica en Deportistas Jóvenes. Exercise and Sport Sciences Reviews. American College of Sports Medicine Series. Vol. 22. pp. 389-443.

Norton K., Olds T. (1996). Antropométric. $1^{\circ}$ Edición. Editorial South Press. Marrickville, Australia.

Pellenc, R.; Costa, I. (2006). Comparación Antropomérica en Futbolistas de Dirente Nivel. http://gse.com/es/entrenamiento-en futbol/articulos/comparacionantropometrica en-futbolistas-de-diferente-nivel-713.

Reilly T, Williams, A.M., Nevill A. \& Franks, A. (2000). A multidisciplinary approach to talent identification in soccer. Journal of Sports Sciences.18; 695-702.

Rienzi E., Mazza J. (1998). Futbolista sudamericano de elite: morfología, análisis del juego y performance. Biosystem Servicio Educativo. Argentina.

Rienzi E, Drust B, Reilly T. Carter JE, Martin A. (2000). Investigation of anthropometric and work-rate profiles of elite South American international soccer players. Journal of Sports Medicine Physical Fitness. 40(2):162-9.

Rivera Sosa, J.M. (2006). Valoración del somatotipo y proporcionalidad de futbolistas universitarios mexicanos respecto a futbolistas profesionales. Revista Internacional de Medicina y Ciencias de la Actividad Física y el Deporte 2006.

Ruiz L., Gutierrez, M., Graupera J., Linaza L., Navarro F. (2001). Desarrollo, comportamiento motor y deporte. Editorial Síntesis S. A. Madrid.

Tomkinson G, Popovic N y Martin M. (2003). Bilateral Simmetry and competitive standart Attained in elite and subelite sport. Journal of sport sciences. 21: (3) 201-211. 
SOTO, V. \& HUERTA, A. (2013). Perfil antropométrico de los países participantes del campeonato sudamericano juvenil femenino de fútbol año 2006. Mot. Hum. 14(1): 29-35.

\section{Dirigir Correspondencia a:}

Alvaro Huerta

Escuela Educación, Carrera de Educación Física

Universidad Santo Tomás, Sede Viña del Mar.

Av. Limonares \# 190, Viña del Mar

CHILE

E-mail: achuertao@yahoo.es

\section{RECIBIDO: 1-05-2013}

ACEPTADO: 29-05-2013 